\title{
Overgeneralization of fear, but not avoidance, following acute stress
}

\author{
Anke Lemmens $^{\mathrm{a}}$, Tom Beckers ${ }^{\mathrm{b}}$, Pauline Dibbets ${ }^{\mathrm{a}}$, Sahaj Kang ${ }^{\mathrm{b}, \mathrm{c}}$, Tom Smeets ${ }^{\mathrm{d}, *}$ \\ ${ }^{\text {a }}$ Faculty of Psychology and Neuroscience, Department of Clinical Psychological Science, Maastricht University, the Netherlands \\ ${ }^{\mathrm{b}}$ Faculty of Psychology and Educational Sciences and Leuven Brain Institute, KU Leuven, Belgium \\ ${ }^{\mathrm{c}}$ Ghent Health Psychology Lab, Ghent University, Belgium \\ ${ }^{\mathrm{d}}$ CoRPS - Center of Research on Psychological disorders and Somatic diseases, Department of Medical and Clinical Psychology, Tilburg School of Social and Behavioral \\ Sciences, Tilburg University, the Netherlands
}

\section{A R T I C L E I N F O}

\section{Keywords:}

Acute stress

MAST

Fear conditioning

Fear generalization

Avoidance generalization

\begin{abstract}
A B S T R A C T
Research has demonstrated the spreading of fear from threat-related stimuli to perceptually similar, but innocuous, stimuli. Less is known, however, about the generalization of avoidance behavior. Given that stress is known to affect learning and memory, we were interested in the effect of acute stress on (over)generalization of fear and avoidance responses. On the first day, one geometrical shape was paired with a mild electrical stimulus $(\mathrm{CS}+)$, whereas another shape was not (CS-). One day later, after participants had been exposed to the Maastricht Acute Stress Test or a control task, generalization of avoidance responses and fear (shock expectancy and skin conductance responses) was tested to a range of perceptual generalization stimuli. Generalization gradients were observed across different outcome measures. Stress enhanced generalization of shock expectancy to the stimulus most similar to the CS + . Our findings confirm that stress can affect the generalization of fear, but further studies are warranted.
\end{abstract}

\section{Introduction}

A key characteristic of many stress- and anxiety-related disorders is the excessive spreading of fear from genuine threat-related stimuli to similar but innocuous cues, a phenomenon called fear overgeneralization (Lissek \& Grillon, 2010; Lissek et al., 2005). For example, overgeneralization of fear has been documented in patients with posttraumatic stress disorder, panic disorder, and generalized anxiety disorder (e.g., Grillon \& Morgan, 1999; Lissek et al., 2010, 2014; Morey et al., 2015). The generalization from threat-associated stimuli to perceptually related stimuli is often investigated in experiments that expose participants to circles of different sizes or colors (e.g., Lissek et al., 2008; Lommen, Engelhard, \& van den Hout, 2010; for a review on fear generalization, see Dymond, Dunsmoor, Vervliet, Roche, \& Hermans, 2015). During conditioning, stimuli at both ends of a size or color continuum are associated with an aversive outcome (e.g., electric stimulus; unconditioned stimulus, US) and the absence of that outcome, respectively, thus serving as the conditioned threat stimulus (CS+) and conditioned safety stimulus (CS-). In a test phase, fear/threat responses (e.g., US-expectancy ratings, fear-potentiated startle (FPS), skin conductance responses (SCR)) to intermediate stimuli on the continuum between $\mathrm{CS}+$ and $\mathrm{CS}-$ are then measured. Fear responses typically generalize to the intermediate stimuli in accordance with their position between the CS + and CS - (Lissek et al., 2008) and the decrease in fear responses as stimuli differ more from the CS+ is less steep in clinical anxiety patients compared to the general population (Dymond et al., 2015).

More recently, the overgeneralization of another type of fear response, namely excessive threat avoidance, has gained attention. Arnaudova, Krypotos, Effting, Kindt, and Beckers (2017) conducted a study that included a differential conditioning phase, where a black or white circle (counterbalanced) was paired with an electric stimulus $(\mathrm{CS}+)$ and the other one was not (CS-). Six intermediate grey-scale stimuli served as generalization stimuli (GSs). Generalization of US expectancies, evaluative ratings, physiological responding (SCR and FPS), and safety behaviors were assessed. In the safety behavior test, participants on certain trials could prevent an expected US by pressing the spacebar. Arnaudova et al. (2017) observed a generalization gradient of behavioral avoidance responses, i.e., the highest percentage of button presses in response to the CS + and the lowest to the CS-, with intermediate responding to the GSs. Generalization of behavioral avoidance has also been investigated by means of a "virtual farmer" task, in which participants could choose to guide a symbolic farmer from his shed to his garden via a short (50\% chance of receiving a shock) or longer road

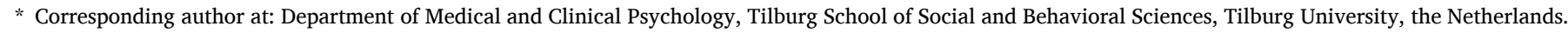

E-mail address: t.smeets@tilburguniversity.edu (T. Smeets).
} 
(avoidance of shock at the cost of poorer performance), while different shapes (CS+, CS -, GSs) appeared in the center of the screen (van Meurs, Wiggert, Wicker, \& Lissek, 2014). It was found that greater generalization of Pavlovian fear responses was associated with overgeneralization of maladaptive avoidance responses and that distress endurance was negatively correlated with rates of maladaptive avoidance to GS stimuli.

Stress affects learning and memory in various ways (Rodrigues, LeDoux, \& Sapolsky, 2009), including fear acquisition and regulation (Raio \& Phelps, 2015; Simon-Kutscher, Wanke, Hiller, \& Schwabe, 2019), learning, consolidation and retrieval of extinction memories (Izquierdo, Wellman, \& Holmes, 2006; Maren \& Holmes, 2016; Wolf, Atsak, De Quervain, Roozendaal, \& Wingenfeld, 2016; Zhou, Kindt, Joëls, \& Krugers, 2011), memory generalization (Krugers et al., 2020; Poulos et al., 2016), and behavioral inhibition and avoidance (Vogel \& Schwabe, 2019). Interestingly, Dunsmoor, Otto, and Phelps (2017) investigated the effect of acute stress on fear generalization in humans in two experiments. All participants underwent a differential threat conditioning procedure in which one tone $(\mathrm{CS}+$ ) was paired with an electric stimulus (US) and another tone predicted safety (CS-). A stress induction procedure (i.e., cold pressor task) or no-stress control task followed immediately (Experiment 1: on Day 1) or $24 \mathrm{~h}$ after threat acquisition (Experiment 2: on Day 2). During a fear generalization test that was administered 15 min after the stress/control task, the CS+, CS - and intermediate tones (GSs) were presented, and US expectancies and SCRs were measured. Results showed that when acute stress was administered following a $24 \mathrm{~h}$ delay (Experiment 2), but not when stress immediately followed acquisition (Experiment 1), participants displayed increased threat generalization in terms of US expectancies and SCRs. Thus, the inability to reliably discriminate between classically conditioned threat cues and generalization stimuli increases over time, such that older fear memories are more likely to result in maladaptive fear generalization. Several explanations for the time-dependency of fear generalization have been proposed, including a loss of memory precision over time (Jasnow, Cullen, \& Riccio, 2012; Riccio, Ackil, \& Burch-Vernon, 1992) and stress-induced changes in the neural circuitry underlying the ability to regulate emotions and to discriminate threat from safety (i.e., impaired hippocampal function: Besnard \& Sahay, 2016; Kheirbek, Klemenhagen, Sahay, \& Hen, 2012; enhanced neural plasticity and hyperactivity in the amygdala: Ghosh \& Chattarji, 2015; Roozendaal, McEwen, \& Chattarji, 2009; Shin \& Liberzon, 2010).

Most research on the effect of stress on fear conditioning has been done in non-humans and has primarily looked at impairments in fear acquisition and extinction rather than at fear generalization (for reviews, see Maren \& Holmes, 2016; Meir Drexler, Merz, Jentsch, \& Wolf, 2019; Raio \& Phelps, 2015; Rodrigues et al., 2009; Wolf et al., 2016; for animal studies, see e.g., Izquierdo et al., 2006; Zhou et al., 2011; for human studies, see e.g., Antov, Melicherová, \& Stockhorst, 2015; Meir Drexler, Hamacher-Dang, \& Wolf, 2017). While this knowledge contributes to a better understanding of stress- and anxiety-related disorders, the effect of acute stress on fear generalization in a standard human fear conditioning procedure has, with the exception of the study by Dunsmoor et al. (2017), remained largely unexplored. Considering the pervasiveness of avoidance symptoms in the clinical presentation of stress- and anxiety-related disorders, the current study not only investigated the effect of stress on fear generalization but also on the generalization of avoidance. More specifically, we examined whether participants who were exposed to acute stress one day after fear acquisition displayed greater overgeneralization of fear responses and increased reliance on avoidant safety behaviors compared to non-stressed controls. Combining the strengths of the experimental designs of Arnaudova et al. (2017) and Dunsmoor et al. (2017), healthy participants underwent a differential threat conditioning procedure with a mild electrical stimulus as US on Day 1. Deviating from a typical generalization procedure (e.g., Lissek et al., 2010) and the study design by Arnaudova et al. (2017), two different geometrical shapes (i.e., a triangle and a cube) that did not lie on a continuum served as CSs and generalization stimuli were constructed by morphing a new stimulus (i. e., a circle) with the two CSs to varying degrees. In doing so, we created two sets of three generalization stimuli that were increasingly dissimilar from one of the CSs yet remained equally dissimilar from the other CS. This provided the opportunity to separate generalization from the CS+ and CS-, thus allowing to distinguish enhanced fear generalization from impaired safety learning generalization. Day 2 started with a stress induction or no-stress control task, followed by an avoidance and generalization test. Outcome measures were US-expectancy ratings, SCR responses, evaluative ratings, and avoidance responses to the CSs and GSs. Exploratorily, all outcome measures were correlated with cortisol reactivity in both the stress and no-stress control group, as it has been shown that altered cortisol reactivity plays an important role in fear inhibition to safety signals (Zuj, Palmer, Malhi, Bryant, \& Felmingham, 2017) and is related to several stress- and anxiety-related disorders (Petrowski et al., 2021; Wichmann, Kirschbaum, Böhme, \& Petrowski, 2017; Zuj et al., 2017). We predicted more generalization in the stress group relative to the no-stress control group, as indicated by increased US expectancy and SCR responses during the fear generalization test and more avoidance to GS stimuli in the stress group relative to the no-stress control group, with a higher percentage of avoidance responses to GSs that were more similar to the $\mathrm{CS}+$.

\section{Method}

\subsection{Participants}

Seventy-one healthy undergraduates (17 male, 54 female) with a mean age of 23.5 years (range $=18-55, S D=5.26$ ) participated in the current study. Based on previous literature (Arnaudova et al., 2017; Dunsmoor et al., 2017), we expected a small to moderate effect $\left(n p^{2}=\right.$ .05 or $f=0.23$ ). $G^{*}$ power analyses with alpha set at .05 and power at .95 for the interaction between group (stress vs. control) and generalization stimuli (four per CS) indicated that a minimum of 44 participants was required. To account for potential attrition and exclusion based on a priori criteria (see data analysis), we recruited and tested until 60 complete datasets (i.e., across day 1 and day 2) were collected. Participants were recruited through advertisements at university billboards, an online research participation environment, and social media. Exclusion criteria were age under 18 and self-reports of cardiovascular or endocrine disorders, history of or current anxiety disorder (including posttraumatic stress disorder), epilepsy, pregnancy or the plan to get pregnant, having a pacemaker, and poor vision (i.e., self-reported vision being (much) worse than others). Before the start of the experiment participants signed a written informed consent form and were randomly assigned to the stress or no-stress control group. After completion of the experiment participants were compensated with a minor financial reward or partial course credit. Test protocols were approved by the Ethics Review Committee Psychology and Neuroscience at Maastricht University (ERCPN-186 06_12_2017) in accordance with the Declaration of Helsinki.

\subsection{Preregistration}

The design of the study and the planned analyses were preregistered on the Open Science Framework at osf.io/8qh6b; see osf.io/ktjaf for an amendment registered prior to data collection. Based on progressive insight, we slightly deviated from the preregistered procedure and analyses. First, we measured cortisol levels as neuroendocrine stress marker, but we did not assess subjective stress levels. Second, $\log (\ln +1)$ transformations were used instead of square root transformations on SCR data, based on expert opinion (P.D.). Third, in contrast to the preregistration we entered the separate GSs as stimuli in our data analyses, in contrast to the averages of the three GSs related to the CS+ and CS-. We did this in order to increase the sensitivity to detect overgeneralization effects. Fourth, we omitted the cortisol responder 
analysis in order to limit the length and thereby increase the readability of our manuscript. Finally, based on the comments by the reviewers, we excluded the CS + from the analyses of the Avoidance Test because the comparison of $\mathrm{CS}+$ and $\mathrm{CS}$ - avoidance was not meaningful given that a majority participants did not avoid the CS + simply because the avoidance button was unavailable for those trials. For the sake of completeness, we reported ineffective avoidance in response to the $\mathrm{CS}+$ in a separate analysis.

\subsection{Materials}

\subsubsection{Fear conditioning protocol}

In the current study we used a fear conditioning protocol based on the procedure by Arnaudova et al. (2017). The protocol consisted of acquisition training on Day 1 and an avoidance and generalization test on Day 2. Fig. 1 presents a schematic overview of the phases and trials of the fear conditioning protocol.

2.3.1.1. CS and US stimuli. A triangle and a cube served as conditioned stimuli (CSs) with stimulus assignment counterbalanced across participants. The unconditioned stimulus (US)

was a 2-ms electrical stimulus administered to the volar surface of the non-dominant forearm. One conditioned stimulus (CS+) was always followed by the US $7.5 \mathrm{~s}$ after onset (i.e., $100 \%$ reinforcement rate), whereas the other conditioned stimulus ( $\mathrm{CS}-$ ) was never followed by the US. One morphing stimulus (MS, circle) and six stimuli with either variously combined properties of the CS+ and MS (GSs+, 3 stimuli) or the CS- and MS (GSs-, 3 stimuli) served as generalization stimuli (see Fig. 2). The CSs, MS, and GSs were all black and superimposed on white square frames and presented in the center of the screen against a black background. The US was delivered via a bipolar bar electrode, covered with conductive gel (K-Y gel, Johnson \& Johnson), and connected to a DS7A Constant Current Stimulator (Digitimer Ltd., Hertfordshire, UK). The protocol on Day 1 commenced with a US calibration procedure in which the electric stimulus was increased incrementally until participants reported that the stimulus was very uncomfortable yet nonpainful.

\subsection{Outcome measures}

\subsubsection{US expectancies}

On each acquisition and generalization trial, US expectancy was measured on an 11-point Likert scale, ranging from -5 (for sure no electric stimulus) to 5 (for sure electric stimulus), presented at the bottom of the screen (i.e., below the geometrical shape). The cursor was located at 0 (uncertain) at the beginning of each trial, regardless of the response given on the previous trial (Arnaudova et al., 2013, 2017). Participants were given $5 \mathrm{~s}$ to move the cursor and confirm their response with a mouse click. If they did not confirm a response, the cursor's last position was recorded.

\subsubsection{Skin conductance responses (SCR)}

During acquisition and the generalization test, SCRs to the CSs, MS, and GSs were measured at the medial phalanges of the index and the middle fingers of the non- dominant hand using two Ag-AgCl electrodes filled with electrolyte gel (Spectra 360, Parker). A Brainvision professional Brainamp ExG Skin Conductor was used that passes the signal to Brain Vision Analyzer 2.0 software. Participants were explicitly instructed not to move the hand to which the electrodes were attached during the computer tasks in order to increase the reliability of the SCR measurements.

\subsubsection{Retrospective evaluative ratings}

Evaluative ratings of CSs, the MS, GSs and the US were collected at the end of the experimental procedure using an 11-point Likert scale ranging from -5 (unpleasant) to 5 (pleasant). The US was evaluated on intensity $(1=$ light, $2=$ moderate, $3=$ intense, $4=$ enormous and $5=$ unbearable) and startlingness $(1=$ not, $2=$ light, $3=$ moderate, $4=$ strong and $5=$ very strong). Finally, participants were asked to rate the perceived effectiveness of the space bar during the avoidance test on a 5point scale ranging from 1 (not at all effective) to 5 (very effective).

2.4.3.1. Acquisition training (day 1). At the start of the acquisition training, participants were instructed that the objects presented on the screen would either be followed by an electrical stimulus or not and that their task was to predict when the electric stimulus would follow. Participants received $10 \mathrm{CS}+$ and $10 \mathrm{CS}-$ trials during the acquisition

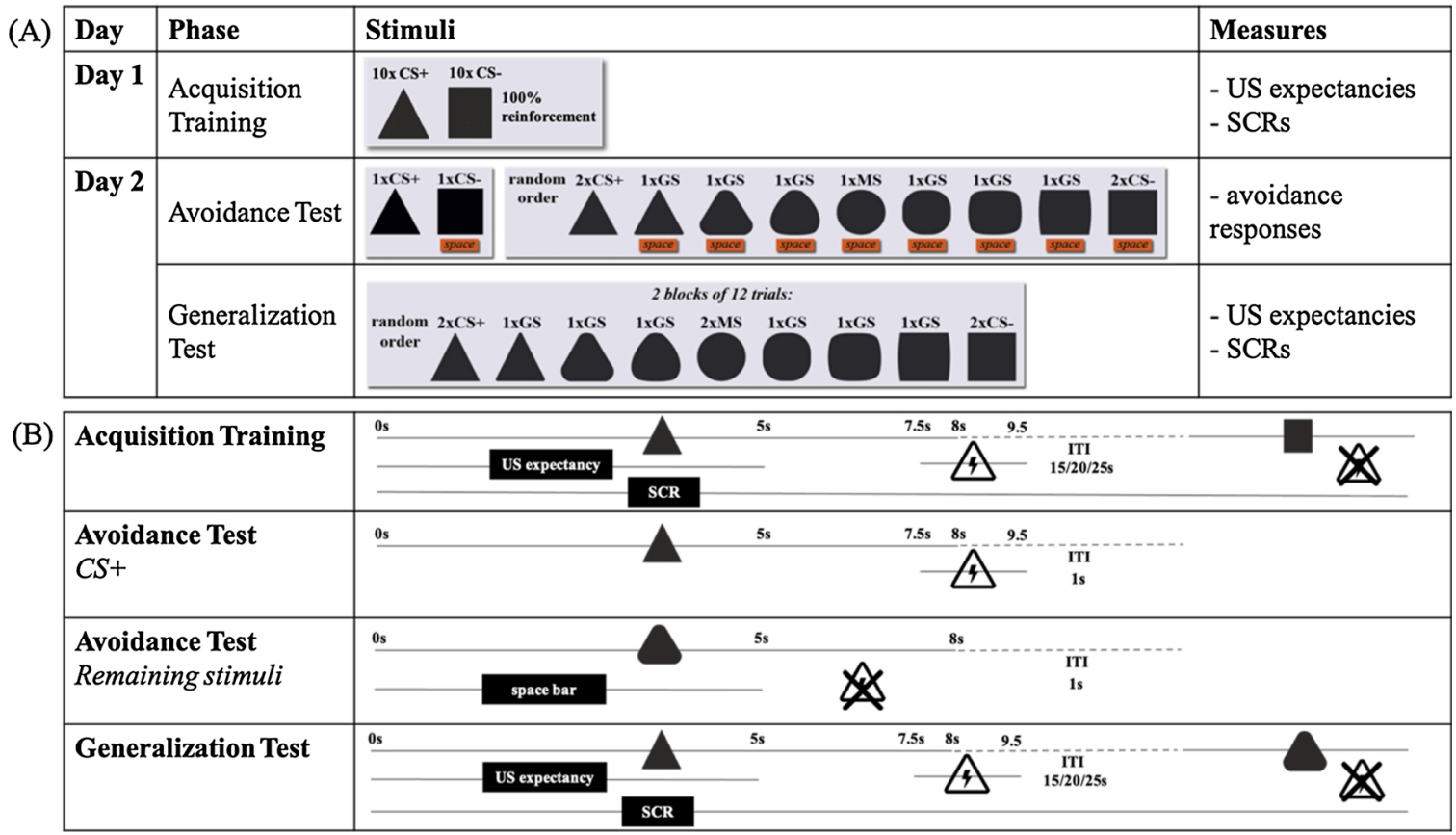

Fig. 1. Overview of the phases (A) and trials (B) of the fear conditioning protocol. 


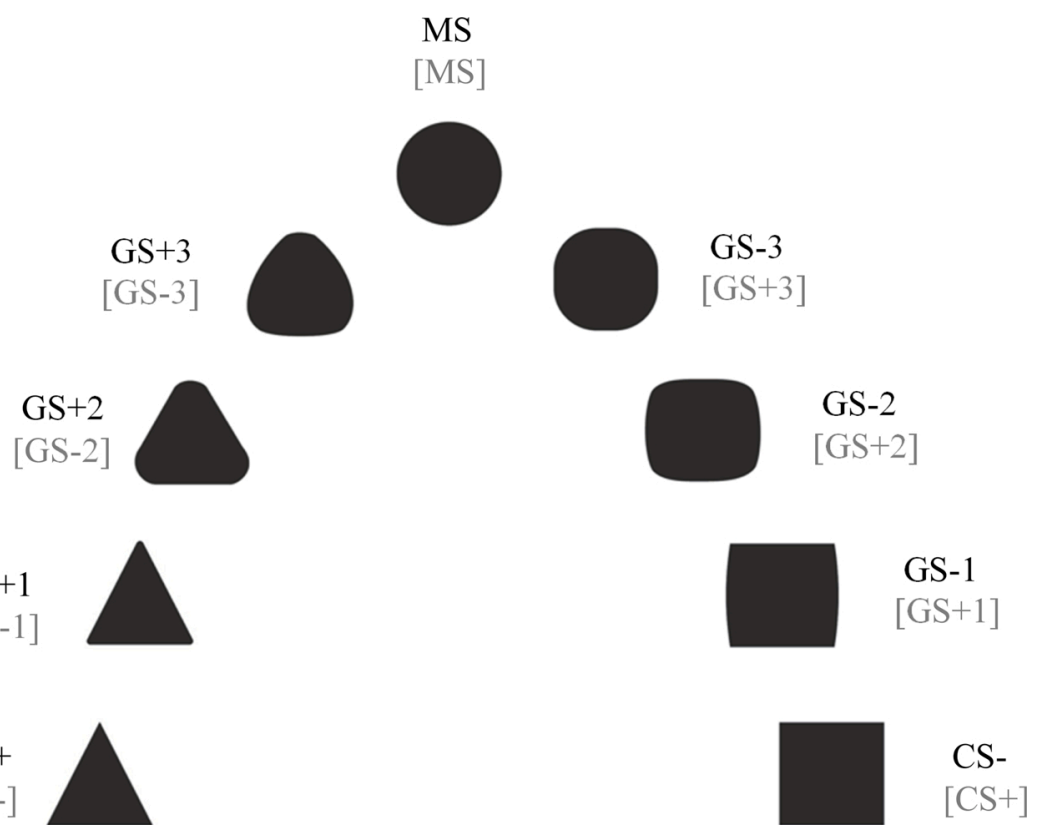

Fig. 2. Conditioned and Generalization Stimuli.

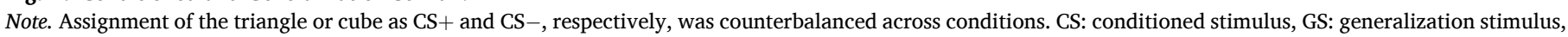
MS: morphing stimulus.

training in random order. Each CS trial had an 8-s duration and trials were separated by an inter-trial interval (ITI) with an average duration of $20 \mathrm{~s}$ (jittered 15, 20 or $25 \mathrm{~s}$ ). The US expectancy scale was only available during the first five seconds of the CS.

2.4.3.2. Avoidance test (day 2). Participants were informed that they could avoid the electric stimulus on some trials, but not on others. More specifically, they were instructed that they could press the avoidance button (i.e., space bar) to prevent the US, but only in case a message appeared under the presented stimulus indicating that the space bar was available. It was stressed that participants should only press the space bar if they believed the stimulus would lead to an electric stimulus and that they should take into consideration what they learned on Day 1. As per Arnaudova et al. (2017), the avoidance test comprised 13 trials: the $\mathrm{CS}+$ and CS - were presented three times, and the MS and all GSs were presented once. The test always started with one presentation of the CS+ and the CS- (i.e., a reminder of what they learned on Day 1), after which the other 11 trials were presented in random order. CS + trials were always followed by the US and no avoidance button was available. However, erroneous button presses were recorded. The avoidance button was available on GS, MS, and CS - trials; irrespective of the avoidance response, these stimuli were never followed by the US. Each avoidance test trial had an 8-s duration and trials were separated by a 1-s ITI. The avoidance button message appeared simultaneously with stimulus onset and was presented underneath the stimuli. Participants had $5 \mathrm{~s}$ to press the space bar.

2.4.3.3. Generalization test (day 2). The generalization test consisted of two blocks of 12 trials: two presentations of the CS+, CS- and MS and one presentation of all GSs per block (i.e., 24 trials in total). Within each block, stimuli were presented randomly. Generalization trials were similar to those in the acquisition training: trials lasted $8 \mathrm{~s}$ and were separated by an ITI with an average duration of $20 \mathrm{~s}$ (jittered 15, 20 or $25 \mathrm{~s}$ ). The CS + was always followed by the US and the GSs, MS, and CSwere never followed by the US. During the generalization test, the avoidance button was not available.

\subsubsection{Stress vs. no-stress control manipulation}

The Maastricht Acute Stress Test (MAST; Smeets et al., 2012; see also: Quaedflieg, Meyer, Van Ruitenbeek, \& Smeets, 2017) was used to induce acute stress in the stress group. The MAST started with a 5-min preparation phase, followed by a 10-min acute stress phase, in which participants were repeatedly exposed to cold pressor stress and mental arithmetic challenges. More specifically, they had to immerse their dominant hand into a bucket with ice-cold water $\left(4^{\circ} \mathrm{C}\right)$ during five trials of different duration (60-90 s). In between those trials, participants had to count backwards as fast and accurately as possible in steps of 17 starting at four different random numbers, e.g., 2043 (for 45-90 s). To induce social evaluative threat, participants were told that they were videotaped during the task and saw themselves on a monitor. Also, they received negative feedback (e.g., "count faster" or "incorrect, start over") when they engaged in the calculations. To increase uncontrollability, participants were told that the order and duration of the hand immersion and mental arithmetic trials would be randomly chosen by the computer.

The no-stress control group completed a validated no-stress control task (Smeets et al., 2012), in which participants had to immerse their hand into lukewarm water $\left(35^{\circ} \mathrm{C}\right)$ and perform a simple counting test (i. e., count aloud from 1 to 25), without being videotaped or receiving negative feedback.

2.4.4.1. Neuroendocrine stress responses. Salivary measurements with synthetic Salivettes (Sarstedt ${ }^{\circledR}$, Etten-Leur, the Netherlands) were obtained in order to measure cortisol reactivity. Participants provided saliva samples at baseline $\left(t_{\text {pre-AcQ }}\right)$ and after the fear acquisition training on Day 1 ( $t_{\text {post-ACQ }}$ ), and before and two times after the MAST on Day $2\left(\mathrm{t}_{\text {pre-MAST }}, \mathrm{t}_{\text {post-MAST1}}, \mathrm{t}_{\text {post-MAST2 }}\right)$. Samples were stored at $-20{ }^{\circ} \mathrm{C}$ until cortisol levels were determined by a commercially available luminescence immune assay kit (IBL, Hamburg, Germany), with mean intra- and inter-assay coefficients of variation below $8 \%$.

\subsubsection{Questionnaires}

2.4.5.1. Distress Tolerance Scale (DTS). The DTS (Simons \& Gaher, 2005) is a 15-item self-report measure of emotional distress tolerance. 
The questionnaire comprises items that reflect one's perceived ability to tolerate emotional distress, subjective appraisal of distress, absorption by negative emotions, and regulation efforts. Items are rated on a 5-point scale, ranging from "strongly agree" to "strongly disagree". Higher scores indicate a higher distress tolerance. The DTS has shown good psychometric properties (Simons \& Gaher, 2005). Cronbach's alpha for the DTS in the current study was $\alpha=.84$.

\subsection{Procedure}

After successful screening for eligibility via email, participants received the following instructions via email: They were kindly invited to eat breakfast but to refrain from eating, smoking, exercising, and drinking anything other than water from two hours before the start of the experiment; and were also asked to take the elevator instead of the stairs (to minimize arousal effects) at the day of the experiment. Testing was constrained to $12.30 \mathrm{~h}-14.30 \mathrm{~h}$ on Day 1 and $14.30 \mathrm{~h}-18.30 \mathrm{~h}$ on Day 2 to minimize morning fluctuations in cortisol levels. The procedure on Day 1 had a duration of approximately 20 min, whereas on Day 2 the procedure took between 45 and $60 \mathrm{~min}$. Fig. 3 displays an overview of the experimental procedure. After reading the information letter and signing for informed consent on Day 1, participants received an overview of the session and saliva sampling procedure instructions. Next, the SCR electrodes and the shock electrodes were attached. Hereafter, participants underwent the US calibration procedure and the first saliva measurement $\left(\mathrm{t}_{\text {pre-ACQ}}\right)$. It was ensured that ten min had passed between entering the lab and the first saliva sample. After the saliva measurement, participants read instructions about the acquisition training on the screen; these were repeated orally by the experimenter to assure that participants had understood the task instructions. After finishing the acquisition training, all electrodes were removed, and the second saliva sample was taken ( $t_{\text {post-ACQ}}$ ).

At the start of the procedure on Day 2, participants received an overview of the second session and the saliva sampling procedure instructions were repeated. Next, they completed the DTS on the computer. At least ten min after the start of the session, the first saliva measurement took place ( $\left.t_{\text {pre-MAST }}\right)$. Participants then underwent either the MAST or no-stress control task and immediately afterwards a second saliva sample was taken ( $t_{\text {post-MAST1 }}$ ). Next, SCR and shock electrodes were attached, and participants performed the avoidance and the generalization tests. Hereafter, all electrodes were removed and participants were asked to complete the retrospective evaluative ratings on the computer. Finally, the last saliva measurement was taken ( $t_{\text {post- }}$ MAST2) and participants were debriefed and compensated for their participation.

\subsection{Data analysis}

Data analyses were performed using SPSS Statistics for Mac, version 25 (SPSS Inc., Chicago, Ill., USA). The data was checked for normality and outliers. If Mauchly's test indicated that the assumption of sphericity had been violated, the degrees of freedom were corrected using Greenhouse-Geisser estimates of sphericity. All reported $p$-values are two-tailed. The standard rejection criterion was set at $p<.05$ throughout. Partial Eta Squared $\left(\eta_{p}^{2}\right)$ values are reported as a measure of effect size for statistically significant results.

Participants were excluded from the analyses if baseline cortisol values on Day 1 or 2 deviated more than three standard deviations from the mean (in both directions). Furthermore, participants were excluded from analyses if they failed to demonstrate differential fear conditioning, as indicated by a lack of discrimination learning observable in US expectancies and SCRs. More specifically, participants had to rate both US expectancy of the CS + above 3 and US expectancy of CS - below - 3 and show higher SCRs to the CS + than to the CS - during the final half of the acquisition training in order to be included in the data analyses. After excluding participants (see Results), a randomization check was performed, comparing demographic variables, DTS scores, shock intensity, and cortisol responses on Day 1 and 2 using Univariate and GLM repeated measures ANOVAs and Fisher's exact tests.

We investigated the effectiveness of the MAST by examining cortisol levels using GLM repeated measures ANOVAs. Cortisol data were logtransformed due to typical skewness of the data. SCRs to the

\section{Day 1}
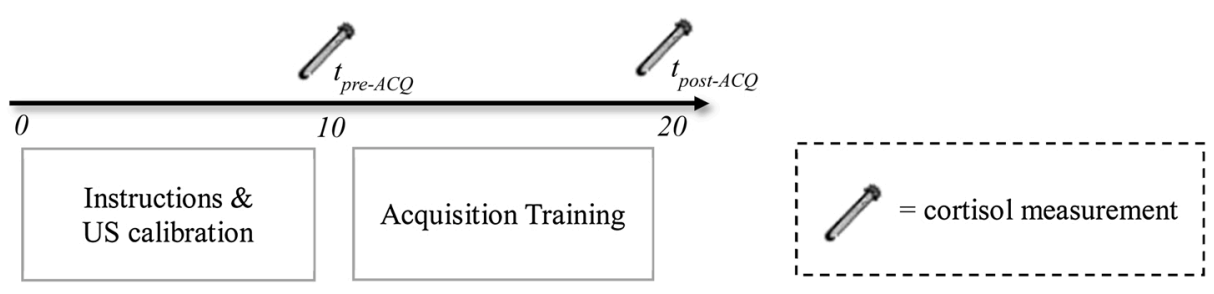

Day 2

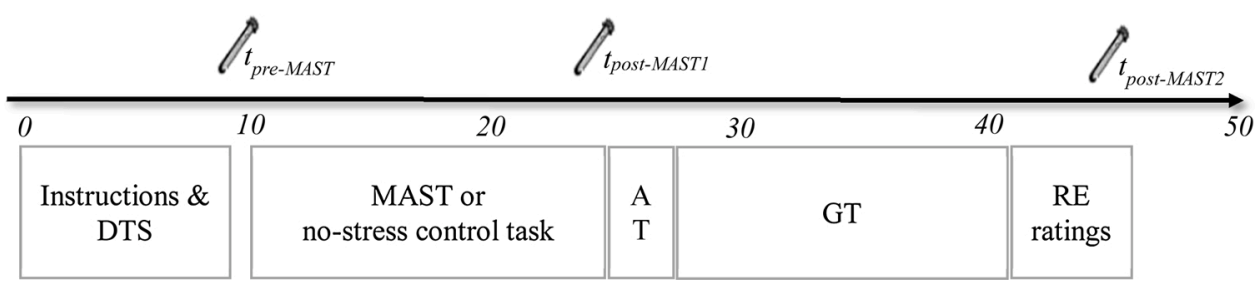

Fig. 3. Overview of the Experimental Procedure.

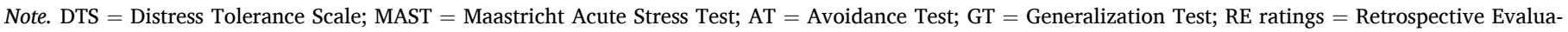
tive ratings. 
conditioned stimuli were analyzed using Ledalab (V3.2.4; http://www. ledalab.de). Data were preprocessed including smoothing (8 G, convolution with a Hanning window) and down sampling to $10 \mathrm{~Hz}$. Artefacts were manually traced and corrected using a spline interpolation. A continuous decomposition analysis was carried out, optimizing the fit and reducing the error of the model (Benedek \& Kaernbach, 2010). Skin conductance responses were level-corrected by subtracting the average skin conductance level of the preceding and succeeding inter-stimulus intervals. Subsequently, event-related activation based on the event-locked markers was calculated by using the largest deflection in conductance between 900 and 4000 ms after stimulus onset (First Interval Response) with a minimum response of .02 $\mu$ s. A $\log (\ln +1)$ transformation was applied to adjust the skewness of the data.

With respect to the acquisition training, we checked for group differences in contingency learning (US expectancy and SCRs) using GLM repeated measures ANOVAs. As preregistered, we calculated mean USexpectancy ratings and SCRs averaged over the final five CS + and $\mathrm{CS}$ - trials of the acquisition training. Regarding the avoidance test, we used a GLM repeated measures ANOVA. The proportion of avoidance responses to the CS - was averaged over three trials. Significant (interaction) effects were followed up with either pairwise comparisons or separate Univariate ANOVAs for all stimuli. Moreover, we investigated the potential impact of a better-safe-than-sorry strategy, and the effect of acute stress on this strategy, by checking avoidance frequencies in response to the $\mathrm{CS}+$ (when no signal was available that participants could avoid the US) using ANOVA, Fisher's exact and chi-square tests. In case participants used the avoidance button on all CS + trials (notwithstanding the absence of an avoidance signal), it is possible that they attributed the occurrence of the US to the ineffectivity of the space bar (i. e., avoidance button) and failed to learn that the US would also occur if they did not perform an avoidance response. Because this may have reduced their avoidance behavior towards the GSs presented in the Avoidance Test, we reran the analyses of the Avoidance Test excluding participants that avoided on all CS + trials. With respect to the generalization test, group differences in generalization gradients (i.e., US expectancy and SCRs) were analyzed using GLM repeated measures ANOVAs. US expectancies and SCRs were averaged over trials and per block. Significant (interaction) effects were followed up with either pairwise comparisons or separate Univariate ANOVAs for all stimuli. Within a block, US expectancies and SCRs to the $\mathrm{CS}+, \mathrm{CS}-$, and MS were averaged across two trials. Group differences in pleasantness of the CS+, CS-, MS and GSs, US pleasantness, US intensity, and US startlingness, and the perceived effectiveness of the avoidance response were tested using Univariate ANOVAs.

Finally, mean DTS scores were correlated to the main dependent variables. Individual cortisol responses on Day 2, defined as peak level post-stress $\left(t_{\text {post-MAST1 }}\right)$ minus baseline $\left(t_{\text {pre-MAST }}\right)$, were correlated to the proportions of avoidance responses to all stimuli, and US expectancies and SCRs to all stimuli in the generalization test within the stress and nostress control groups.

\section{Results}

\subsection{Data availability}

The data files and data analysis syntax of the results reported here can be obtained through the Open Science Framework (osf.io/jnd8f).

\subsection{Included sample}

As data of 11 participants was incomplete either due to technical and logistic problems $(n=3)$ or to drop-out $(n=8), 71$ participants were tested to achieve a dataset of 60 participants with data from day 1 and day 2 completed (as preregistered). Of the 60 full datasets, another 10 were excluded from the analyses because they did not meet the predefined learning criteria. More specifically, two participants were excluded because no discrimination learning in US expectancies was observed, and nine participants failed to show differential learning in SCRs (note that one participant was excluded based on both criteria). Hence, the total sample consisted of $N=50$ participants. Groups did not differ in gender ratio $(p=.40$, two-sided Fisher's exact test) or age $(F(1$, $48)=.14, p=.71)$. There was also no difference between the groups in electric stimulus intensity (mA) after US calibration $(F(1,48)=.14, p=$ .71). Groups did not differ in baseline cortisol levels on Day $1(F(1,48)=$ $3.61 p=.06)$ and there were no significant increases in cortisol levels on Day $1(F s(1,48)<.006, p s>.94)$. Thus, there were no differences in how stressed both groups were in terms of cortisol prior to the acquisition training and the acquisition training per se was not stressful. More importantly, groups did not differ in cortisol levels before the MAST on Day $2(F(1,48)=1.23, p=.27)$. There was a significant difference in distress tolerance $(F(1,48)=4.93, p=.03)$, indicated by higher mean DTS scores $(M=3.68)$ in the stress group compared to the no-stress control group $(M=3.33)$. However, we did not include mean DTS scores as a covariate in our analyses, as DTS scores did not correlate with our main outcome measures (see Correlational analyses).

\subsection{Stress manipulation}

The effect of the stress induction procedure on salivary cortisol levels was assessed using GLM repeated measures ANOVAs with Group (stress vs. no-stress control) as between-subjects variable and Time ( $t_{\text {pre-MAST, }}$ $\mathrm{t}_{\text {post-MAST1 }}, \mathrm{t}_{\text {post-MAST2 }}$ ) as within-subjects variable. A significant Time*Group interaction was observed $\left(F(2,96)=11.58, p<.001, \eta_{p}^{2}=.19\right)$. Post-hoc analyses of Group per timepoint revealed that cortisol levels were not different between the stress and no-stress control groups before the MAST ( $\mathrm{t}_{\text {pre-MAST: }} F(1,48)=1.23, p=.27$ ) or directly at the end of the MAST ( $\mathrm{t}_{\text {post-MAST1}}: F(1,48)=.37, p=.55$ ), but as expected differed between groups approximately 20 min after the stress induction ( $\mathrm{t}_{\text {post- }}$ MAST2: $F(1,48)=4.81, p=.03)$, with higher cortisol levels in the stress group.

\subsection{Acquisition training}

GLM repeated measures ANOVAs with Stimulus (CS+ vs. CS-) as within-subjects factor and Group (stress vs. no-stress control) as between-subjects factor demonstrated no Group (interaction) effects (Fs $<3.45$, ps $>$.07). Not surprisingly given our predefined learning criteria, the Stimulus effect was significant (US expectancy: $F(1,48)=$ 139456.61, $p<.001, \eta_{p}^{2}>$.99; SCRs: $F(1,48)=75.39, p<.001, \eta_{p}^{2}=$ .61 ), as can be seen in Fig. 4.

\subsection{Avoidance test}

A GLM repeated measures ANOVA with Stimulus (GS + 1, GS + 2, GS +3 , MS, GS-3, GS-2, GS-1, CS-) as within-subjects factor and Group (stress vs. no-stress control) as between-subjects factor demonstrated a significant Stimulus effect $\left(F(3.35,160.87)=32.22, p<.001, \eta_{p}^{2}=.40\right)$. The main effect of Group and the Group difference in the effect of Stimulus were not significant $(F s<1.34, p s>.25$ ). Bonferroni-corrected pairwise comparisons yielded significant differences in the proportions of avoidance responses between the GS +1 and all other stimuli ( $p s<$ $.001)$, between the GS +2 and the GS-3, GS-1 and CS $-(p s<.04)$, and between the GS +3 and the GS-3, GS- 1 , and CS - ( $p s<.03$ ). The proportions of avoidance to all stimuli (except $\mathrm{CS}+$ ) for the stress and nostress control groups are displayed in Fig. 5.

In order to investigate the occurence of a better-safe-than-sorry strategy, we investigated how many participants pressed the space bar in response to the $\mathrm{CS}+$ in the absence of a signal that the US could be avoided on that trial. It was found that four participants pressed the space bar on all CS + trials (i.e., employed a better-safe-than-sorry strategy), one participant pressed the space bar two out of three CS+ trials, and 14 participants pressed the space bar only once in response to 
A

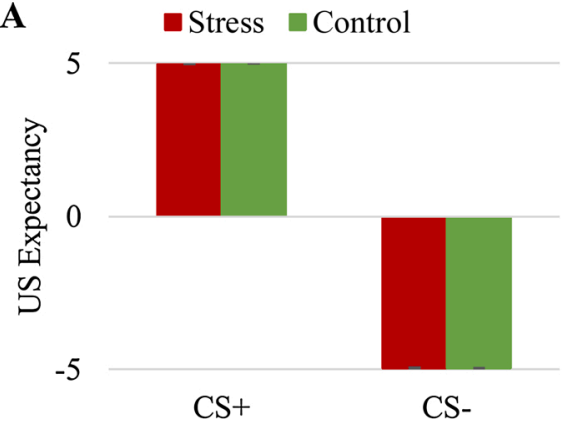

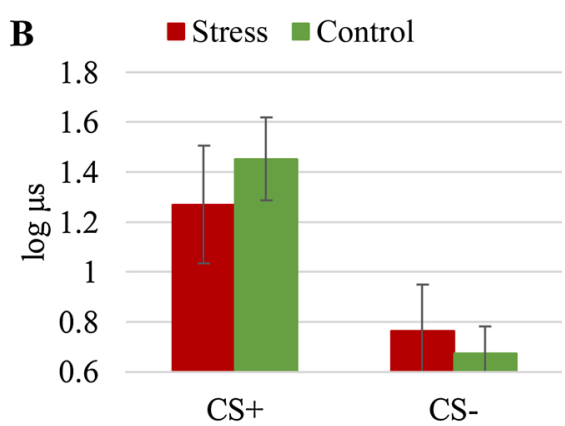

Fig. 4. Results of the Acquisition Training.

Note. (A) US-expectancy ratings ( $\pm \mathrm{SE}$ ) and (B) log-transformed SCRs $( \pm \mathrm{SE})$ to the $\mathrm{CS}+$ and $\mathrm{CS}-$.

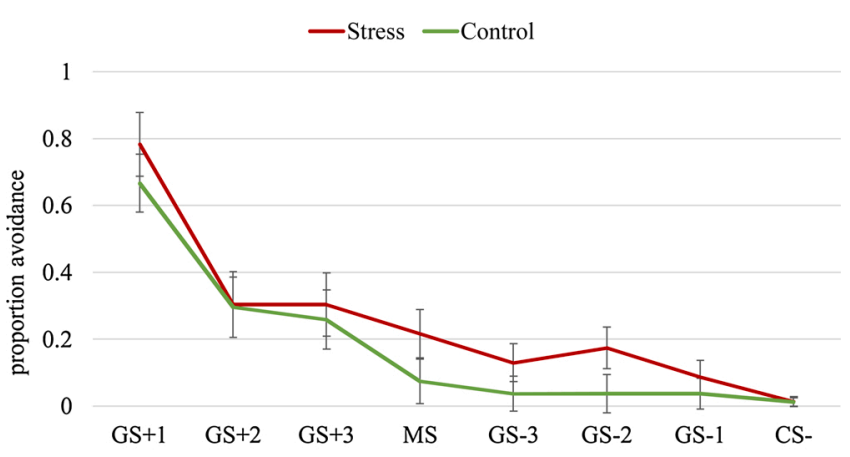

$-0.2$

Fig. 5. Results of Avoidance Test.

Note. Proportion of avoidance responses $( \pm \mathrm{SE})$ to all stimuli. Avoidance to CS + is not presented, because there was no avoidance signal on $\mathrm{CS}+$ trials.

a CS + . The stress and control group did not differ in the use of a bettersafe-than sorry strategy ( $p>.99$, two-sided Fisher's exact test), their use of the space bar on one or more CS + trials $\left(\chi^{2}(1)=1.20, p=.27\right)$, or the proportion of avoidance responses to the $\operatorname{CS}+(F(1,48)=1.78, p=.19)$. This indicates that acute stress did not affect avoidance behaviors towards the $\mathrm{CS}+$. When re-running the analyses of the Avoidance Test excluding the four participants that avoided on all CS + trials, highly similar results were obtained.

\subsection{Generalization test}

A GLM repeated measures ANOVA on US-expectancy ratings with Stimulus (CS+, GS + 1, GS + 2, GS + 3, MS, GS-3, GS-2, GS-1, CS-) as within-subjects factor and Group (stress vs. no-stress control) as between-subjects factor demonstrated a significant Stimulus* Group effect $\left(F(3.00,143.75)=2.80, p=.04, \eta_{p}^{2}=.06\right)$. Separate ANOVAs per stimulus showed that the stress and no-stress control groups differed significantly in US-expectancy ratings for the GS $+1(F(1,48)=5.92, p$ $=.02)$, MS $(F(1,48)=6.38, p=.01$, and GS-1 $(F(1,48)=4.88, p=.03)$. US-expectancy ratings for the remaining stimuli were not significantly different between groups ( $F \mathrm{~s}<2.11$, $p \mathrm{~s}>.15$ ). Similar analyses were performed on SCR data. The repeated measures ANOVA yielded a significant Stimulus effect only $\left(F(5.92,284.28)=12.36, p<.001, \eta_{p}^{2}=\right.$ .21 ; Group (interaction) effects: $F \mathrm{~s}<1.62$, $p \mathrm{~s}>.21$ ). Bonferronicorrected pairwise comparisons yielded significant differences in SCRs between the CS + and all other stimuli $(p s<.002)$ and between the GS + 1 and the GS +2 and MS ( $p s<.02$ ). Fig. 6 shows the US-expectancy ratings and SCRs to all stimuli during the generalization test.

\subsection{Retrospective evaluative ratings}

The stress and no-stress control groups were compared on pleasantness of all stimuli, US pleasantness, US intensity, US startlingness, and perceived effectiveness of the avoidance response using Univariate ANOVAs with Group as between-subjects factor. Groups did not significantly differ in their pleasantness ratings of any of the stimuli (Fs $<3.04, p s>.09$, see Fig. 7). There were also no significant group differences in ratings of US pleasantness $(F(1,48)=1.20, p=.28 ; M=$ $-3.69, S D=1.01)$, US intensity $(F(1,48)=2.33, p=.13 ; M=2.86, S D=$ $.61)$, US startlingness $(F(1,48)=.004, p=.95 ; M=2.86, S D=1.01)$, or perceived effectiveness of the avoidance response $(F(1,48)=.69, p=$ $.41 ; M=2.72, S D=1.51$ ).

\subsection{Correlational analyses}

We calculated correlations between DTS scores on the one hand and US-expectancy ratings and SCRs during acquisition, proportions of avoidance responses to all stimuli (avoidance test), and US-expectancy ratings and SCRs during the generalization test on the other hand. DTS scores only correlated significantly with US expectancy of GS-3 during the generalization test $(r(50)=-.28, p=.048)$. No other correlations were significant ( $p s>.06)$. Given multiple testing, the significant correlation should be interpreted with great caution.

We also calculated correlations between individual cortisol responses on Day 2 (i.e., $t_{\text {post-MAST1 }}$ minus $t_{\text {pre-MAST }}$ ) on the one hand and the percentage of avoidance responses to CSs, MS, and GSs in the avoidance test and US expectancy and SCRs to the CSs, MS, and GSs in the generalization test within the stress and no-stress control groups on the other hand. Individual cortisol responses did not correlate with any of the outcome variables in either group (all $p s>.06$ ).

\section{Discussion}

The aim of the current study was to examine whether participants who were exposed to acute stress one day after fear acquisition displayed greater overgeneralization of fear responses and increased reliance on avoidant safety behaviors compared to non-stressed controls. We predicted more generalization in the stress relative to the no-stress control group, as indicated by increased US expectancy and SCR responses during the fear generalization test and a higher percentage of avoidance responses to GSs that were related to the CS+ in the stress group relative to the no-stress control group. After successful fear acquisition in terms of US expectancies and SCRs, a typical generalization gradient was observed in US-expectancy ratings and SCRs during the generalization test. Moreover, acute stress enhanced generalization of US expectancy to the stimulus that was most similar to the CS + . This enhancing effect of stress was not observed in SCR data. Finally, although we found generalization of avoidance responses to the GS +1 , no effect of acute stress was observed.

The generalization gradients observed in US expectancies and SCRs converge with findings of earlier studies that demonstrated highest fear responses towards the $\mathrm{CS}+$, lowest to the $\mathrm{CS}-$, and intermediate 

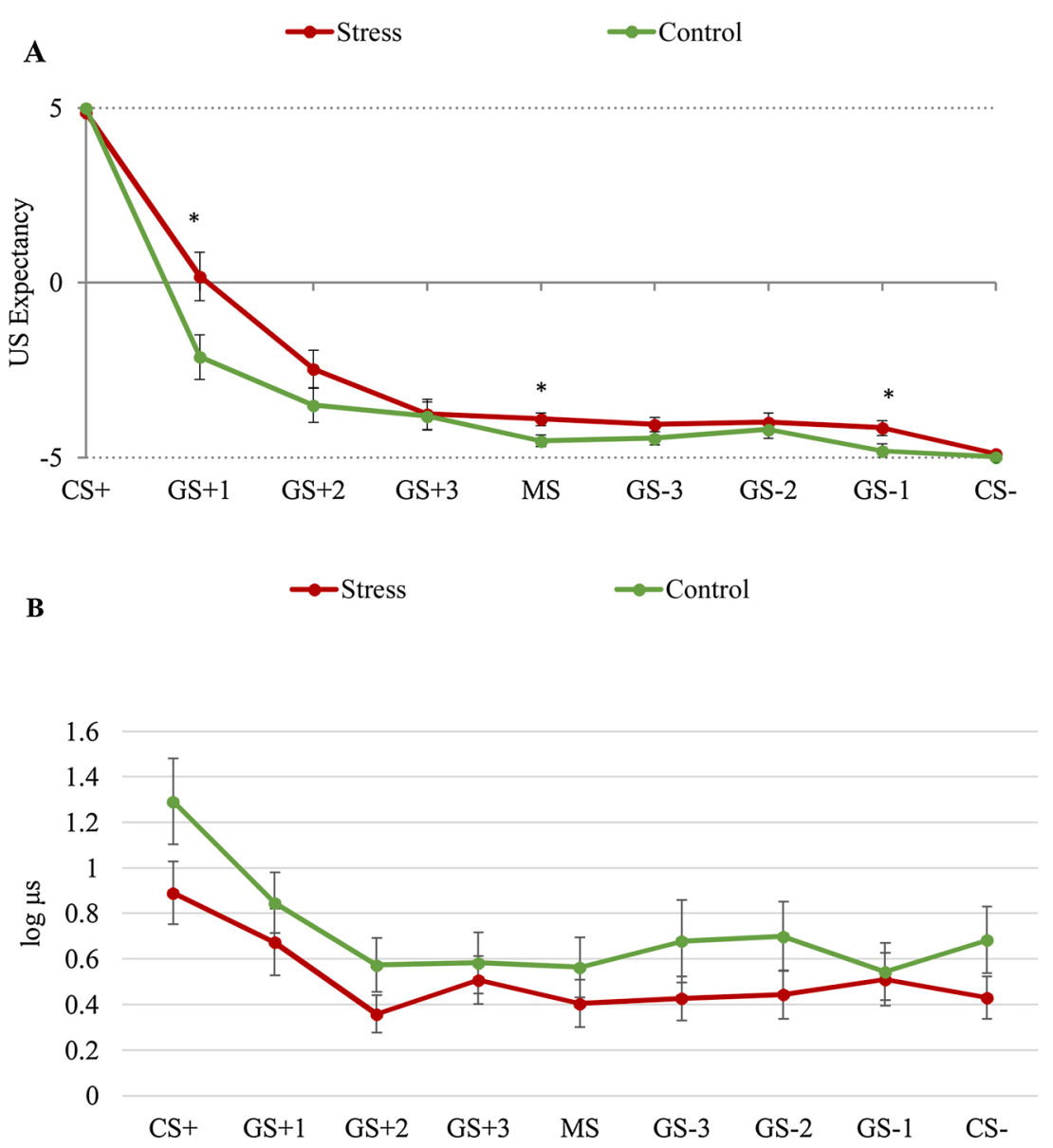

Fig. 6. Results of the Generalization Test.

Note. (A) US-expectancy ratings ( \pm SE) and (B) log-transformed SCRs $( \pm$ SE) to all stimuli.

* Significant Group effects are marked, $p<.05$.

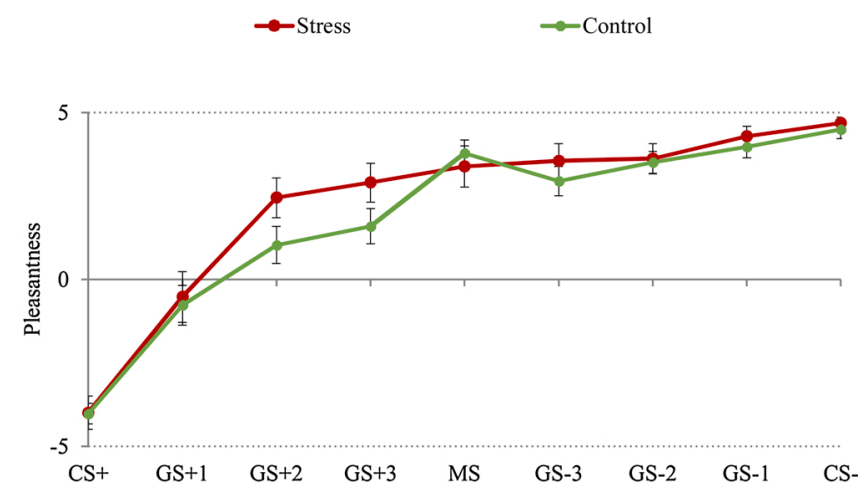

Fig. 7. Retrospective pleasantness ratings of the CSs, MS, and GSs ( \pm SE).

responses to GSs (e.g., Arnaudova et al., 2017; Dunsmoor et al., 2017; Lissek et al., 2008). The generalization gradient was also observable in pleasantness ratings of the stimuli and in the proportion of avoidance responses towards the GSs. This latter finding corresponds with the results of earlier studies that found the highest proportion of avoidance responses towards a stimulus that most closely resembled the CS+ (Arnaudova et al., 2017; van Meurs et al., 2014). The replication of the generalization gradient across studies and across different types of measures (i.e., automatic, physiological measures like SCRs and subjective and controlled measures such as US expectancy, avoidance responses and evaluative ratings) indicates that fear generalization is a robust phenomenon (see also Dymond et al., 2015).
In line with Dunsmoor et al. (2017), we found that acute stress resulted in higher US expectancy towards the stimulus that most closely resembled the $\mathrm{CS}+$. Several neuroscientific explanations for the effect of stress on fear generalization have been put forward. One of them is that stress leads to impaired hippocampal function (Besnard \& Sahay, 2016; Kheirbek et al., 2012). This theory suggests that stress impairs the pattern separation function of the dentate gyrus. In line with this theory, it has been demonstrated that glucocorticoids modify properties of memory-encoding neuronal ensembles in the dentate gyrus in mice (Krugers et al., 2020). As a consequence, it is more difficult to discriminate between past threats and the presently encountered stimulus, resulting in generalization of fear responses (Dunsmoor et al., 2017). Another explanation lies in the fact that stress leads to enhanced neural plasticity and hyperactivity in the amygdala (Ghosh \& Chattarji, 2015; Roozendaal et al., 2009; Shin \& Liberzon, 2010). For example, Ghosh and Chattarji (2015) found alterations in the response of lateral amygdala neurons in rats as a result of increased shock intensity. These alterations changed the balance of activity toward a greater proportion of generalizing over cue-specific neurons. In humans, this theory is supported by the fact that patients with posttraumatic stress disorder show hyperactivity in the amygdala to cues that resemble a learned threat (Morey et al., 2015).

A novel aspect of the current study was the use of generalization stimuli that did not lie on a continuum from CS + to CS - but were constructed by morphing a new stimulus (i.e., a circle) with the two CSs (i.e., triangle and cube) to varying degrees. As such, while the three GSs that were formed by morphing the triangle with the circle are increasingly dissimilar from the triangle, all three are equally dissimilar from 
the cube (and vice versa for the morphed cube-circle GSs versus the triangle). This provides the opportunity to examine generalization gradients from the CS+ and CS - separately, thus allowing to distinguish enhanced fear generalization from impaired safety learning generalization. The results of the Generalization Test were not only indicative of enhanced fear generalization (discussed above), but also of impaired safety learning. We found that acute stress enhanced US expectancy towards a stimulus that most closely resembled the CS- and to a novel stimulus with the highest threat ambiguity (i.e., MS). Although these novel findings require replication in further studies, enhanced responding to the GS-1 as a result of acute stress coincides with another preliminary finding, showing a positive correlation between cortisol responding after a stress induction and the startle response to the CSduring acquisition learning, indicating impaired safety learning (Klinke, Fiedler, Lange, \& Andreatta, 2020). Since stress is both a catalyst and cardinal symptom of anxiety disorders, our finding is also in line with studies showing greater fear responding towards a safe stimulus in high trait anxious individuals (Gazendam, Kamphuis, \& Kindt, 2013) and clinical anxiety patients (e.g., Grillon \& Morgan, 1999; Hermann, Ziegler, Birbaumer, \& Flor, 2002; Jovanovic et al., 2009; Lissek et al., 2009; Orr et al., 2000; Peri, Ben-Shakhar, Orr, \& Shalev, 2000), and with the theory that pathological anxiety is associated with a failure to inhibit a fear response in the presence of safety signals (Davis, Falls, \& Gewirtz, 2000). Enhanced responding to the MS also aligns well with studies showing greater fear responding and overgeneralization of fear to stimuli with high threat ambiguity in high trait anxious individuals (Boddez et al., 2012; Chan \& Lovibond, 1996; Wong \& Lovibond, 2018).

We did not replicate the effect of acute stress on SCRs (Dunsmoor et al., 2017) and we did not observe an effect of stress on avoidance responses. Furthermore, it is important to note that our results are indicative of fear generalization, but not of overgeneralization per se. Participants only showed generalization of fear and avoidance responses towards the stimulus that most closely resembled the CS+. Moreover, stress led to increased US expectancy of the GS +1 in comparison to the no-stress control group, however, no excessive fear responding towards GSs that were less similar to the CS + was observed. Although Dunsmoor et al. (2017) found higher US expectancies in the stress group not only in response to the tone that most closely resembled the CS+ (i.e., GS4) but also to the GS3 during the first block of the generalization test, this pattern was not stable across measures (i.e., SCRs). First, the lack of an overgeneralization effect could be explained by the use of healthy participants. Most studies using a non-clinical sample demonstrated fear generalization and not overgeneralization (e.g., Arnaudova et al., 2017; Dunsmoor et al., 2017; Lissek et al., 2008; van Meurs et al., 2014). Overgeneralization, however, has been observed in clinical groups or groups at risk of developing clinical anxiety (panic disorder: Lissek et al., 2010; generalized anxiety disorder: Lissek et al., 2014; posttraumatic stress disorder: Lissek \& Grillon, 2012; high neuroticism: Lommen et al., 2010). So, it might be the case that overgeneralization is a phenomenon that distinguishes pathological anxiety from normal anxiety. Second, it is worth bearing in mind that in contrast to the typical generalization procedure used in previous research (e.g., Lissek et al., 2008), we created generalization stimuli by morphing a new stimulus with the two CSs to varying degrees. Morphing of the CS + and CS - with a third novel stimulus may have resulted in GSs that were perceptually easier to distinguish than, for example, circles with different shades of grey or different sizes. Especially in a population of healthy controls in which overgeneralization is less discernible, combining the two distinct CSs might have made it more difficult to observe overgeneralization effects.

A few limitations of the current study are worth mentioning. First, the current study relied on a sample of healthy students. This makes it difficult to translate findings to clinical populations. Another limitation of the sample used in the current study is that it potentially suffers from selection bias. The advertisement informed participants about the electrical stimulation during the experiment. Hence, it is possible that individuals with a combination of some specific personality traits (e.g., low trait anxiety, high distress tolerance, high sensation seeking) are overrepresented in our sample (Karos, Alleva, \& Peters, 2018). Third, when designing the experiment, a decision about the order of the tests had to be made. As the effect of stress on generalization of avoidance was never studied before, we decided to start with the avoidance test, followed by the generalization test. In order to minimize interference, the avoidance test included only one trial per GS. However, it cannot be ruled out that the information learned about the GSs during the avoidance test influenced fear responses during the generalization test. Fourth, our main analyses resulted in several $p$-values that fall between $p$ $=.03$ and $p=.05$. It is worth noting that when power is high (i.e., in this study the sample size was based on a power of $95 \%$ ), it should be unlikely for multiple $p$-values to fall within this range when the alternative hypothesis is true (Lakens, 2015). This may somewhat undermine the robustness of our findings. Fifth, our sample size calculation was conducted for and based on US-expectancy ratings. However, there is more variability in skin conductance responses and skin conductance data are usually skewed (Lonsdorf et al., 2017), limiting the usefulness of sample size calculations on the basis of normal approximations (Cundill \& Alexander, 2015). Hence, our analysis of the SCR data might be underpowered.

Overall, our results contribute to the conclusion that fear generalization is a robust phenomenon. We observed generalization gradients in US expectancies, SCRs, avoidance responses, and pleasantness ratings. Moreover, acute stress enhanced generalization of US expectancy to the stimulus that was most similar to the $\mathrm{CS}+$. No effect of acute stress on SCRs and avoidant responses was observed. As stress- and anxietyrelated psychopathologies involve the frequent experience of acute and chronic stress, it is important to study the effect of stress on different aspects of fear conditioning, including fear overgeneralization, which is assumed to be an important developmental and maintaining factor in clinical anxiety (Dymond et al., 2015). The current study is one of the first efforts to unravel the effect of stress on fear and avoidance generalization, however, more studies are needed to examine the robustness and potential boundary conditions of the effects of acute stress on fear and avoidance generalization in healthy as well as (sub)clinical populations.

\section{Funding}

This work was supported by the Dutch Research Council (Nederlandse Organisatie voor Wetenschappelijk Onderzoek, NWO; \#452-14003) to T.S.

\section{Author contributions}

Anke Lemmens: Conceptualization, Methodology, Formal Analysis, Visualization, Writing - Original Draft; Pauline Dibbets: Conceptualization, Methodology, Writing - Review \& Editing; Tom Beckers: Conceptualization, Methodology, Writing - Review \& Editing; Sahaj Kang: Conceptualization, Methodology, Investigation, Writing - Review \& Editing; Tom Smeets: Conceptualization, Methodology, Formal Analysis, Visualization, Supervision, Writing - Review \& Editing.

\section{Declaration of Competing Interest}

The authors report no declarations of interest.

\section{Acknowledgment}

We thank Inna Arnaudova for providing us with the programming code from Arnaudova et al. (2017). 


\section{References}

Antov, M. I., Melicherová, U., \& Stockhorst, U. (2015). Cold pressor test improves fear extinction in healthy men. Psychoneuroendocrinology, 54, 54-59.

Arnaudova, I., Krypotos, A. M., Effting, M., Boddez, Y., Kindt, M., \& Beckers, T. (2013) Individual differences in discriminatory fear learning under conditions of ambiguity: A vulnerability factor for anxiety disorders? Frontiers in Psychology, 4, 298.

Arnaudova, I., Krypotos, A. M., Effting, M., Kindt, M., \& Beckers, T. (2017). Fearing shades of grey: Individual differences in fear responding towards generalisation stimuli. Cognition and Emotion, 31(6), 1181-1196.

Benedek, M., \& Kaernbach, C. (2010). A continuous measure of phasic electrodermal activity. Journal of Neuroscience Methods, 190(1), 80-91.

Besnard, A., \& Sahay, A. (2016). Adult hippocampal neurogenesis, fear generalization, and stress. Neuropsychopharmacology, 41(1), 24-44.

Boddez, Y., Vervliet, B., Baeyens, F., Lauwers, S., Hermans, D., \& Beckers, T. (2012). Expectancy bias in a selective conditioning procedure: Trait anxiety increases the threat value of a blocked stimulus. Journal of Behavior Therapy and Experimental Psychiatry, 43(2), 832-837.

Chan, C. K., \& Lovibond, P. F. (1996). Expectancy bias in trait anxiety. Journal of Abnormal Psychology, 105(4), 637-647.

Cundill, B., \& Alexander, N. D. (2015). Sample size calculations for skewed distributions. BMC Medical Research Methodology, 15, 28.

Davis, M., Falls, W. A., \& Gewirtz, J. (2000). Neural systems involved in fear inhibition: Extinction and conditioned inhibition. In M. Myslobodsky, \& I. Weiner (Eds.), Contemporary issues in modeling psychopathology (pp. 113-142).

Dunsmoor, J. E., Otto, A. R., \& Phelps, E. A. (2017). Stress promotes generalization of older but not recent threat memories. Proceedings of the National Academy of Sciences, 114(34), 9218-9223.

Dymond, S., Dunsmoor, J. E., Vervliet, B., Roche, B., \& Hermans, D. (2015). Fear Generalization in humans: Systematic review and implications for anxiety disorder research. Behavior Therapy, 46(5), 561-582.

Gazendam, F. J., Kamphuis, J. H., \& Kindt, M. (2013). Deficient safety learning characterizes high trait anxious individuals. Biological Psychology, 92(2), 342-352.

Ghosh, S., \& Chattarji, S. (2015). Neuronal encoding of the switch from specific to generalized fear. Nature Neuroscience, 18(1), 112-120.

Grillon, C., \& Morgan, C. A. (1999). Fear-potentiated startle conditioning to explicit and contextual cues in Gulf War veterans with posttraumatic stress disorder. Journal of Abnormal Psychology, 108(1), 134-142.

Hermann, C., Ziegler, S., Birbaumer, N., \& Flor, H. (2002). Psychophysiological and subjective indicators of aversive pavlovian conditioning in generalized social phobia. Biological Psychiatry, 52(4), 328-337.

Izquierdo, A., Wellman, C. L., \& Holmes, A. (2006). Brief uncontrollable stress causes dendritic retraction in infralimbic cortex and resistance to fear extinction in mice. Journal of Neuroscience, 26(21), 5733-5738.

Jasnow, A. M., Cullen, P. K., \& Riccio, D. C. (2012). Remembering another aspect of forgetting. Frontiers in Psychology, 3, 175.

Jovanovic, T., Norrholm, S. D., Fennell, J. E., Keyes, M., Fiallos, A. M., Myers, K. M., Duncan, E. J. (2009). Posttraumatic stress disorder may be associated with impaired fear inhibition: Relation to symptom severity. Psychiatry Research, 167(1-2), $151-160$.

Karos, K., Alleva, J. M., \& Peters, M. L. (2018). Pain, please: An investigation of sampling bias in pain research. The Journal of Pain, 19(7), 787-796.

Kheirbek, M. A., Klemenhagen, K. C., Sahay, A., \& Hen, R. (2012). Neurogenesis and generalization: A new approach to stratify and treat anxiety disorders. Nature Neuroscience, 15(12), 1613-1620.

Klinke, C. M., Fiedler, D., Lange, M. D., \& Andreatta, M. (2020). Evidence for impaired extinction learning in humans after distal stress exposure. Neurobiology of Learning and Memory, 167, Article 107127.

Krugers, H., Lesuis, S., Xiong, H., van der Loo, R., Kushner, S., \& Lucassen, P. (2020). Glucocorticoids promote fear generalization by altering memory encoding neuronal ensembles (Abstract). Biological Psychiatry, 87(9), S30-S31.

Lakens, D. (2015). How a p-value between 0.04-0.05 equals a p-value between 0.16-017. March 20. The 20\% Statistician http://daniellakens.blogspot.com/2015/03/how-pvalue-between-004-005-equals-p.html.

Lissek, S., \& Grillon, C. (2010). Overgeneralization of conditioned fear in the anxiety disorders: Putative memorial mechanisms. Zeitschrift für Psychologie, 218(2), 146-148.

Lissek, S., \& Grillon, C. (2012). Learning models of PTSD. In J. G. Beck, \& D. M. Sloan (Eds.), The Oxford handbook of traumatic stress disorders. New York, NY: Oxford University Press.

Lissek, S., Biggs, A. L., Rabin, S. J., Cornwell, B. R., Alvarez, R. P., Pine, D. S., \& Grillon, C. (2008). Generalization of conditioned fear-potentiated startle in humans: Experimental validation and clinical relevance. Behaviour Research and Therapy, 46 (5), 678-687.

Lissek, S., Kaczkurkin, A. N., Rabin, S., Geraci, M., Pine, D. S., \& Grillon, C. (2014). Generalized anxiety disorder is associated with overgeneralization of classically conditioned fear. Biological Psychiatry, 75(11), 909-915.

Lissek, S., Powers, A. S., McClure, E. B., Phelps, E. A., Woldehawariat, G., Grillon, C., \& Pine, D. S. (2005). Classical fear conditioning in the anxiety disorders: A metaanalysis. Behaviour Research and Therapy, 43(11), 1391-1424.
Lissek, S., Rabin, S., Heller, R. E., Lukenbaugh, D., Geraci, M., Pine, D. S., \& Grillon, C. (2010). Overgeneralization of conditioned fear as a pathogenic marker of panic disorder. American Journal of Psychiatry, 167(1), 47-55.

Lissek, S., Rabin, S. J., McDowell, D. J., Dvir, S., Bradford, D. E., Geraci, M., ... Grillon, C. (2009). Impaired discriminative fear-conditioning resulting from elevated fear responding to learned safety cues among individuals with panic disorder. Behaviour Research and Therapy, 47(2), 111-118.

Lommen, M. J., Engelhard, I. M., \& van den Hout, M. A. (2010). Neuroticism and avoidance of ambiguous stimuli: Better safe than sorry? Personality and Individual Differences, 49(8), 1001-1006.

Lonsdorf, T. B., Menz, M. M., Andreatta, M., Fullana, M. A., Golkar, A., Haaker, J., Merz, C. J. (2017). Don't fear 'fear conditioning': Methodological considerations for the design and analysis of studies on human fear acquisition, extinction, and return of fear. Neuroscience \& Biobehavioral Reviews, 77, 247-285.

Maren, S., \& Holmes, A. (2016). Stress and fear extinction. Neuropsychopharmacology, 41 (1), 58-79.

Meir Drexler, S., Hamacher-Dang, T. C., \& Wolf, O. T. (2017). Stress before extinction learning enhances and generalizes extinction memory in a predictive learning task. Neurobiology of Learning and Memory, 141, 143-149.

Meir Drexler, S., Merz, C. J., Jentsch, V. L., \& Wolf, O. T. (2019). How stress and glucocorticoids timing-dependently affect extinction and relapse. Neuroscience \& Biobehavioral Reviews, 98, 145-153.

Morey, R. A., Dunsmoor, J. E., Haswell, C. C., Brown, V. M., Vora, A., Weiner, J., LaBar, K. S. (2015). Fear learning circuitry is biased toward generalization of fear associations in posttraumatic stress disorder. Translational Psychiatry, 5(12), e700.

Orr, S. P., Metzger, L. J., Lasko, N. B., Macklin, M. L., Peri, T., \& Pitman, R. K. (2000). De novo conditioning in trauma-exposed individuals with and without posttraumatic stress disorder. Journal of Abnormal Psychology, 109(2), 290-298.

Peri, T., Ben-Shakhar, G., Orr, S. P., \& Shalev, A. Y. (2000). Psychophysiologic assessment of aversive conditioning in posttraumatic stress disorder. Biological Psychiatry, 47(6), 512-519.

Petrowski, K., Schmalbach, I., Strunk, A., Hoyer, J., Kirschbaum, C., \& Joraschky, P. (2021). Cortisol reactivity in social anxiety disorder: A highly standardized and controlled study. Psychoneuroendocrinology, 123, Article 104913.

Poulos, A. M., Mehta, N., Lu, B., Amir, D., Livingston, B., Santarelli, A., \& Fanselow, M. S. (2016). Conditioning-and time-dependent increases in context fear and generalization. Learning \& Memory, 23(7), 379-385.

Quaedflieg, C. W. E. M., Meyer, T., Van Ruitenbeek, P., \& Smeets, T. (2017). Examining habituation and sensitization across repetitive laboratory stress inductions using the MAST. Psychoneuroendocrinology, 77, 175-181.

Raio, C. M., \& Phelps, E. A. (2015). The influence of acute stress on the regulation of conditioned fear. Neurobiology of Stress, 1, 134-146.

Riccio, D. C., Ackil, J. K., \& Burch-Vernon, A. (1992). Forgetting of stimulus attributes: Methodological implications for assessing associative phenomena. Psychological Bulletin, 112(3), 433-445.

Rodrigues, S. M., LeDoux, J. E., \& Sapolsky, R. M. (2009). The influence of stress hormones on fear circuitry. Annual Review of Neuroscience, 32, 289-313.

Roozendaal, B., McEwen, B. S., \& Chattarji, S. (2009). Stress, memory and the amygdala. Nature Reviews Neuroscience, 10(6), 423-433.

Shin, L. M., \& Liberzon, I. (2010). The neurocircuitry of fear, stress, and anxiety disorders. Neuropsychopharmacology, 35(1), 169-191.

Simon-Kutscher, K., Wanke, N., Hiller, C., \& Schwabe, L. (2019). Fear without context: Acute stress modulates the balance of cue-dependent and contextual fear learning. Psychological Science, 30(8), 1123-1135.

Simons, J. S., \& Gaher, R. M. (2005). The Distress Tolerance Scale: development and validation of a self-report measure. Motivation and Emotion, 29(2), 83-102.

Smeets, T., Cornelisse, S., Quaedflieg, C. W. E. M., Meyer, T., Jelicic, M., \& Merckelbach, H. (2012). Introducing the Maastricht Acute Stress Test (MAST): A quick and non-invasive approach to elicit robust autonomic and glucocorticoid stress responses. Psychoneuroendocrinology, 37(12), 1998-2008.

van Meurs, B., Wiggert, N., Wicker, I., \& Lissek, S. (2014). Maladaptive behavioral consequences of conditioned fear-generalization: A pronounced, yet sparsely studied, feature of anxiety pathology. Behaviour Research and Therapy, 57, 29-37.

Vogel, S., \& Schwabe, L. (2019). Stress, aggression, and the balance of approach and avoidance. Psychoneuroendocrinology, 103, 137-146.

Wichmann, S., Kirschbaum, C., Böhme, C., \& Petrowski, K. (2017). Cortisol stress response in post-traumatic stress disorder, panic disorder, and major depressive disorder patients. Psychoneuroendocrinology, 83, 135-141.

Wolf, O. T., Atsak, P., De Quervain, D. J., Roozendaal, B., \& Wingenfeld, K. (2016). Stress and memory: A selective review on recent developments in the understanding of stress hormone effects on memory and their clinical relevance. Journal of Neuroendocrinology, 28, 1-8.

Wong, A. H., \& Lovibond, P. F. (2018). Excessive generalisation of conditioned fear in trait anxious individuals under ambiguity. Behaviour Research and Therapy, 107, 53-63.

Zhou, M., Kindt, M., Joëls, M., \& Krugers, H. J. (2011). Blocking mineralocorticoid receptors prior to retrieval reduces contextual fear memory in mice. PloS One, 6(10), 26220.

Zuj, D. V., Palmer, M. A., Malhi, G. S., Bryant, R. A., \& Felmingham, K. L. (2017). Endogenous cortisol reactivity moderates the relationship between fear inhibition to safety signals and posttraumatic stress disorder symptoms. Psychoneuroendocrinology, 78, 14-21. 